\title{
ETV5 overexpression promotes progression of esophageal squamous carcinoma by upregulating SKA1 and TRPV2
}

\section{Mingchuang Sun}

Shanghai Dongfang Hospital: Tongji University Dongfang Hospital

\section{Kang Fang}

Shanghai Dongfang Hospital: Tongji University Dongfang Hospital

Zhaoxing Li

Shanghai Dongfang Hospital: Tongji University Dongfang Hospital

\section{Yuan Chu}

Shanghai Dongfang Hospital: Tongji University Dongfang Hospital

\section{Aiping Xu}

Shanghai Dongfang Hospital: Tongji University Dongfang Hospital

\section{Ziying Zhao}

Shanghai Dongfang Hospital: Tongji University Dongfang Hospital

\section{Zhuyun Leng}

Shanghai Dongfang Hospital: Tongji University Dongfang Hospital

\section{Yunwei Zhang}

Shanghai Dongfang Hospital: Tongji University Dongfang Hospital

\section{Zehua Zhang}

Shanghai Dongfang Hospital: Tongji University Dongfang Hospital

\section{Li Zhang}

Shanghai Dongfang Hospital: Tongji University Dongfang Hospital

\section{Tao Chen}

Shanghai Dongfang Hospital: Tongji University Dongfang Hospital

Meidong Xu (D 1800512@tongji.edu.cn)

Shanghai Dongfang Hospital: Tongji University Dongfang Hospital https://orcid.org/0000-0002-72195826

\section{Research Article}

Keywords: Esophageal squamous cell carcinoma, ETV5, SKA1, TRPV2, MMPs, metastasis

Posted Date: August 24th, 2021 
DOl: https://doi.org/10.21203/rs.3.rs-820720/v1

License: (c) (1) This work is licensed under a Creative Commons Attribution 4.0 International License. Read Full License 


\section{Abstract \\ Background}

Esophageal squamous cell carcinoma (ESCC) is notorious for the rapid progression especially early tumor metastasis due to the unclear mechanism. Recently, ETV5 attracts much attention for its potential role as an oncogenic transcription factor involved in multiple cancers. However, no one reported the mechanism behind the association between ETV5 expression and Esophageal squamous cell carcinoma (ESCC) progression. Therefore, in this study, we aimed to investigate the role of ETV5 in ESCC.

\section{Methods}

The Cancer Genome Atlas (TCGA) and GEO database was used to explore ETV5 expression in ESCC. ECA109, KYSE150 and TE1 cell lines were used in the experiments. The Cell Counting Kit 8 (CCK8), migration, invasion, wound healing assays were exerted to evaluate proliferation, migration and invasion of ESCC cells. RNA sequencing was performed to find downstream genes regulated by ETV5. Real-time PCR, Western blotting, Chromatin immunoprecipitation (CHIP) and Dual luciferase reporter assays were used to assess the underlying mechanism. Immunohistochemistry was used to evaluate the relationship between ETV5 expression, clinicopathological parameters and patient prognosis. Tumor metastasis was investigated in BALB/c nude mice.

\section{Results}

ETV5 was observed upregulated in ESCC both from online database and our ESCC tissues and ETV5 was associated with tumor staging. Knockdown of ETV5 or its downstream genes SKA1 and TRPV2 significantly suppress ESCC cells migration and invasion, respectively. Additionally, in vivo study showed knockdown of ETV5 inhibited tumor metastasis. Further experiments unveiled ETV5 could transcriptionally upregulate the expression of SKA1 and TRPV2 and further activate MMPs in ESCC progression.

\section{Conclusion}

ETV5 promoted metastasis of ESCC by activating MMPs through augmenting the transcription of SKA1 and TRPV2. ETV5 was likely to be a novel diagnostic marker and therapeutic target in ESCC treatment.

\section{Background}

Esophageal cancer is a global health problem with an overall 5-year less than $20 \%[1]$. So far, China occupied the most significant proportion in esophageal cancer cases and deaths worldwide and esophageal squamous cell carcinoma (ESCC) was the main histological type [2, 3]. ESCC is notorious for 
its rapid progression, especially early tumor metastasis and lacking effective treatment. Therefore, much attention should be paid to explore the biological behavior, diagnosis markers, and approach targets of ESCC.

As one of the largest families of signal-dependent transcription factors, the E26 transformation-specific (ETS) family comprises 28 homologs. The PEA3 subset of the ETS family, contains three factors: ETV1/ER81, ETV4/PEA3/E1AF, and ETV5/ERM[4]. Recently, ETV5 attracts much attention for its important role as an oncogenic transcription factor in multiple cancer types and its involvement in multiple biological processes. For example, ETV5 is related to EMT in papillary thyroid cancer[5]. ETV5 accelerates tumor growth by promoting cell cycle G1/S transition in colorectal cancer[6]. Additionally, ETV5 is linked to the maintenance of cancer stem cell (CSC) phenotype in breast cancer[7]. Yet to date, the role of ETV5 played in ESCC is still unknown. Through TCGA and GEO databases, we found ETV5 was significantly elevated in ESCC, but the true function of ETV5 in ESCC has never been studied. Thus, to evaluate and identify the role of ETV5 in ESCC development and progression, further study is needed.

In this study, we investigated the expression and function of ETV5 in ESCC and further identified its downstream targets by employing both in vitro and in vivo assays for the first time. The results revealed the oncogenic role of ETV5 in ESCC progression.

\section{Materials And Methods}

\section{Data collection}

The Cancer Genome Atlas (TCGA) is a large-scale cancer genomics program, and it has molecularly characterized 33 primary cancer types comprising esophageal squamous cell carcinoma. GEO is a public genomics platform composited of array- and sequence-based data. Using UALCAN and Oncomine, the expression of ETV5 in ESCC was investigated from TCGA and GEO database.

\section{Cell culture}

We purchased human ESCC cell lines, ECA109, KYSE150 and TE1 from the Institute of Biochemistry and Cell Biology of the CAS (Shanghai, China). All cell lines were cultured in DMEM medium (GIBCO) supplemented with $10 \% \mathrm{FBS}$ (GIBCO) in an incubator containing $5 \% \mathrm{CO} 2$ at $37^{\circ} \mathrm{C}$.

\section{Cell transfection}

For transient transfection, cells were seed in 6-well plates, 100 pmol siRNA-ETV5 or siRNA-SKA1 or siRNATRPV2 (GenePharma, Shanghai, China) was transduced into each well. Real-time quantitative polymerase chain reaction (RT-qPCR) and western blotting were applied to detect transfection efficiency at $48 \mathrm{~h}$ after transfection. For stable transfection, lentivirus vectors that encode a shRNA targeting ETV5 or shRNA non-targeting control were used to transfect ECA109 cells following the manufacturer's instruction (Genechem, Shanghai, China). In brief, cells were seed in 6-well plates, medium containing 
viral fluid but without serum was added when the cell density reached $30 \%$ and replaced with complete medium 24h later. The transfection efficiency of each vector was detected via western blotting.

\section{CCK8 assay}

For evaluating the proliferation capacity of ESCC cells, the Cell Counting Kit 8 (CCK8) assay was used. We planted $1 \times 10^{3}$ cells/well in the 96-well plates, and $10 \mu$ l CCK8 reagent was used to mix cells in every well respectively after cells being incubated for $0,24,48,72$ and $96 \mathrm{~h}$. After $4 \mathrm{~h}$ of incubation, we measured the absorbance of cells at $450 \mathrm{~nm}$ by using a microplate reader.

\section{Migration and invasion assay}

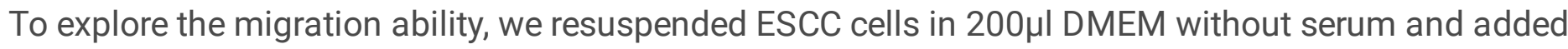
them in upper chamber of the transwell device, with $5 \times 10^{4}$ cells/well. We then added $600 \mu l$ complete medium into the lower chamber as the chemical attractants. After incubation for $48 \mathrm{~h}$ at $37^{\circ} \mathrm{C}$, cells on the lower surface of the non-coated membrane were fixed by $4 \%$ paraformaldehyde and then stained by Giemsa. Images from five representative fields of each membrane were taken by using a light microscope (100x). The number of migratory cells was counted and the relative migration rate can be calculated. Invasion assay was similar to migration assay, but with the difference that $100 \mu \mathrm{l}$ of $200 \mu \mathrm{g} / \mathrm{ml}$ diluted Matrigel matrix (Corning, 356234) was carefully added to the center of each transwell insert and incubated at $37^{\circ} \mathrm{C}$ for 1 hour to form a gel before cells were plated.

\section{Wound healing assay}

The ECA109 and KYSE150 cells underwent a culturing process in six-well plates and when the cells reached $80 \%$ confluence, the monolayers were scraped by the tip of a $200 \mu l$ pipette, and cells continued to be cultured in DMEM free of serum. At 0,24 and $48 \mathrm{~h}$ after scratch, cell migration was photographed by a light microscope (100x). Image $\mathrm{J}$ was used to calculate the closure. The formulas are: average scratch width $=$ scratch gap area/length; the relative cell migration rate $=$ (the scratch width at Oh-scratch width after culture)/the scratch width at $0 \mathrm{~h} \times 100 \%$.

\section{Real-time PCR assay and RNA sequencing}

Total RNAs were extracted from cells by using Trizol solution, according to the instruction of the manufacturer. We used Nanodrop2000 spectrophotometer to measure the RNA concentrations and synthesized complementary DNA (cDNA) from RNA by using a PrimeScript RT reagent kit (Takara, Japan). TaqMan real-time PCR assays for ETV5, SKA1 and TRPV2 were applied following the instruction of Takara Bio. The relevant primers were summarized in supplementary table 1. All reactions, including the no-template controls were run in triplicate. After the reactions, the CT values were determined using fixed threshold settings. Library preparation for RNA sequencing was conducted. Generally, $1 \mu \mathrm{g}$ highquality RNA was used, and sequencing was carried out by HiSeq2500 (Illumina Inc., San Diego, CA) at Genechem Biotechnology (Shanghai) Co., Ltd.

\section{Western blotting analysis}


After being washed by PBS, all kinds of cell lines were harvested in RIPA buffer. The protein concentrations were measured using Bradford assay. Samples were separated on 8-12\% SDS-PAGE gels and then transferred to PVDF membrane (Millipore, USA) and probed with primary antibodies specific for ETV5 (ab102010, Abcam), MMP2 (ab92536, Abcam), MMP9 (ab76003, Abcam), SKA1 (bs-7846R, Bioss) and TRPV2 (bs-10297R, Bioss). $\beta$-Actin and GAPDH were used as loading controls for western blots.

\section{Chromatin immunoprecipitation (CHIP) assay}

A CHIP assay was performed using an Upstate Biotechnology kit. Briefly, we successively subjected cells to the procedures containing DNA-protein cross-linking, disruption of membrane and cytosol. Samples were digested by MNase and sonicated and then precipitated with antibody against transcriptional factor ETV5. Quantitative real-time PCR was used to measure the amount of bound DNA. According to the relative amount of input and the IgG ratio, the enrichment value was calculated. The primers covering ETV5 binding site of SKA1 and TRPV2 gene promoter region were summarized in supplementary table 1.

\section{Dual luciferase reporter assay}

ESCC cells were seeded into 12 -well plates at a density of $2.5 \times 10^{4}$ cells/well. The reporter plasmid containing wild SKA1 or TRPV2 promoter and mutant SKA1 or TRPV2 promoter at the binding site, was transfected into cells, respectively. The cells were also transfected with Renilla luciferase reporter plasmids for signal normalization. After $24 \mathrm{~h}$, the luciferase activity was measured by the Dual Luciferase Assay System Kit (Promega, Madison, WI, USA).

\section{Immunohistochemistry (IHC)}

This study was approved by the institutional review board of East Hospital, Tongji University. ESCC and corresponding healthy esophageal mucosa (CHEM) tissues of 98 patients were collected. After being fixed by Formalin and embedded by paraffin, tissue sections were cut to $4 \mu \mathrm{m}$ thickness and then placed in xylene and graded alcohols for deparaffinization and hydration. We performed heat-induced antigen retrieval in EDTA (PH 8.0) buffer for 15 minutes by using a microwave oven. To reduce nonspecific staining, we performed blocking with $10 \%$ goat serum. After the specific primary anti-ETV5 (ab102010, Abcam) was dropped onto the sections and incubated overnight at $4{ }^{\circ} \mathrm{C}$, the slides were counterstained with light hematoxylin, dehydrated, and cover-slipped.

\section{Animal studies}

This in vivo study was approved by the animal care and use committee of Tongji University. 20 female $\mathrm{BALB} / \mathrm{c}$ nude mice ( 6 weeks old) were used for animal studies. The animals were randomly divided into 2 groups (control and treated groups, 10 mice per group). ECA109 cells were treated with stable transfection. After cell harvest, cells were resuspended and injected into the tail vein of each mouse ( $2 \times 10^{6}$ viable ECA109 cells/mouse). At 6 weeks, the lung metastasis was monitored. According to the AVMA Guidelines for the Euthanasia of Animals, we performed intraperitoneal injection of a three-fold dose of barbiturates to euthanize all the mice. After that, lungs were removed, and the lung colonization number was counted. Serial sections of lung tissue were stained with hematoxylin and eosin. 


\section{Statistical analysis}

The statistical analysis was performed by SPSS 19.0 (IBM, Armonk, NY, USA). All the experiments were carried out repeatedly three times. Differences between groups were calculated using Student's t-test, Chisquare test, or Fisher's exact test. The $p$ value $<0.05$ was considered with statistical significance.

\section{Results}

\section{ETV5 expression is upregulated in esophageal squamous cell carcinoma.}

To investigate the expression of ETV5 in ESCC, we initially analyzed the online data from TCGA and GEO by using UALCAN and Oncomine, respectively. ETV5 is significantly upregulated in ESCC, not only supported by the RNA-sequencing data from TCGA, but also validated by data from Oncomine. The expression of ETV5 is higher in ESCC cells than that in normal esophageal epithelial cells. Besides, IHC analysis showed that upregulation of ETV 5 was also found in our ESCC tissues. Moreover, by analyzing the correlation of clinicopathologic parameters with ETV5 level in ESCC tissues, we found that ETV5 protein level was higher in Stage III-IV cancers than in Stage 0-II cancers.

\section{ETV5 silencing suppresses migration and invasion but not the proliferation of ESCC cells.}

To further study how ETV 5 affects the functions of ESCC cells, CCK8, migration, invasion and wound healing assays were performed. The expression of ETV5 was knocked down by transfecting specific siRNA targeting ETV5, indicated by real-time PCR and western blot results. The results of CCK8 assays indicated that knockdown of ETV5 did not decrease the cell proliferation in ECA109 and KYSE150 cell lines. However, the migration and wound healing assays results indicated that cell migration in these two cell lines was obviously suppressed when ETV5 was knocked down. Additionally, the results of invasion assay also showed that knockdown of ETV5 evidently decreased cell invasion in these two cell lines.

\section{ETV5 transcriptionally regulates SKA1 and TRPV2}

In order to identify the molecular mechanism underlying the ETV5-mediated increase in cancer progression, RNA-seq analysis was performed to compare protein-coding transcripts levels in ECA109, KYSE150 and TE1 cells treated with or without siETV5. With $P<0.05$ and $F C>2$ as cutoff values, 100 downregulated genes in siETV5 transfected cells were found. Among the 100 genes, SKA1 and TRPV2 attracted our attention because though the mechanism was unclear, its' over-expression in ESCC had been reported $[8,9]$. We performed real-time PCR and western blot validation in ESCC cells after knockdown ETV5. In ECA109 and KYSE150 cells transfected with siETV5, SKA1 and TRPV2 mRNA were significantly downregulated. Considering ETV5 is a transcript factor, we proposed that ETV5 may exert its functions by regulating the transcription of SKA1 and TRPV2. CHIP assay was done to verify the binding site of ETV5 in the SKA1 and TRPV2. Besides, luciferase assays demonstrated that ETV 5 could increase SKA1 and TRPV2 transcriptional activation. Moreover, MMP2 and MMP9, which were reported to be 
downstream of SKA1 or TRPV2 in many other cancers, were found to be downregulated in ESCC cells when SKA1 or TRPV2 was knocked down by siRNAs.

\section{SKA1 or TRPV2 silencing suppresses migration and invasion of ESCC cells.}

To explore the function of SKA1 and TRPV2 in ESCC cells, migration, invasion and wound healing assays were performed. The expressions of SKA1 and TRPV2 were knocked down by transfecting specific siRNA, respectively, indicated by real-time PCR and western blot results. The migration and wound healing assays results indicated that cell migration in these two cell lines was suppressed when whatever SKA1 or TRPV2 was knocked down. It could also be easily detected that cell invasion in these two cell lines was decreased when whatever SKA1 or TRPV2 was knocked down from the invasion assays.

\section{Effect of ETV5 on the Vivo metastasis of ECA109}

We further studied the ETV5 effect on cancer metastasis by mice modes. In the tail vein injection nude mouse model, the average number of lung metastasis per mouse was significantly reduced in shETV5transfected ECA109 cells compared with the control cells. Histological analysis demonstrated that the metastatic nodules formed by shETV5-transfected cells were smaller than those formed by controlled cells.

\section{Discussion}

ESCC always has a poor prognosis because of its inconspicuous symptoms and metastasis in the early stage, which limits effective therapies to a great extent. Thus, understanding its distinct biological process and find novel diagnostic markers and therapeutic targets is imperative. In this study, we found that the expression level of ETV5 was higher in ESCC than that in the normal esophagus, both from tissue and cell aspects, which was validated by analyzing the data from TCGA and GEO database. Furthermore, the expression level of ETV5 was correlated to tumor stage and prognosis from the IHC and the analysis of online data. These results demonstrated that ETV5 might act as an oncogene similar to ETS-1[10] and ETV4[11] in ESCC. We further applied in vitro and in vivo studies and found that knockdown of ETV5 significantly suppressed ESCC migration and invasion, which indicated that ETV5 could augment ESCC metastasis.

Considering ETV5 is a transcription factor, ETV5 should regulate some downstream targets during ESCC progression. We further exerted RNA-seq analysis and found two interesting genes SKA1 and TRPV2. Though they were ever reported to be overexpressed in ESCC, the concrete function and underlying mechanism are still obscure. Kinetochore-associated complex includes SKA1, SKA2 and SKA3. This complex plays a leading role in stabilizing the attachment of spindle microtubules to kinetochores and maintaining the metaphase plate during mitosis $[12,13]$. Acting as an oncogene in many cancer types, SKA1 contributes to multiple biological behaviors, including cell circle, EMT, and Wnt/ $\beta$-catenin pathways $[14,15]$. Thus, its proper function in ESCC is worth exploring. Transient receptor potential Vanilloid family is a non-selective calcium-permeable channel and acts as a cellular sensor for heat, 
stretch and osmosis[16]. Recently, it has raised much attention that TRPV2 acts as a cancer biomarker and potential therapeutic target for many cancer types[17], but the specific function in ESCC is not very clear. Herein, we speculated that SKA1 and TRPV2 could promote ESCC progression, transcriptionally regulated by ETV5. Firstly, the results of in vitro studies verified the oncogenic roles of SKA1 and TRPV2 in migration and invasion of ESCC cells, so they could also augment ESCC metastasis. Additionally, the results of Real-time PCR and western blotting analysis indicated ETV5 could regulate the expression of SKA1 and TRPV2. Moreover, the CHIP assays and luciferase assays results further told us that ETV5 could directly bind to the promotor of SKA1 and TRPV2 and activate their transcription.

As mentioned above, ETV5 could promote ESCC metastasis by directly regulating SKA1 and TRPV2, but the involved biological process was not clear. MMPs are crucial agents responsible for extracellular matrix degradation, and they are always considered important factors accounting for tumor metastasis. Besides, they are common downstream factors of ETS protein, especially the PEA3 subfamily[18]. Of MMPs, MMP2 and MMP9 were the main members presented to be regulated by SKA1 and TRPV2 in some cancers, such as human adenoid cystic carcinoma, non-small cell lung cancer and prostate cancer[19-21]. Thus, these two MMPs may also take part in ESCC metastasis. Through Real-time PCR and western blotting analysis, we found the expression level of MMP2 and MMP9 are in accordance with that of SKA1 and TRPV2, which indicated both SKA1 and TRPV2 could promote ESCC metastasis by cotargeting MMP2 and MMP9. In this study, we demonstrated that ETV5 was overexpressed in ESCC cells, and correlated to poor prognosis. ETV5 promoted metastasis of ESCC by activating MMPs through augmenting the transcription of SKA1 and TRPV2. These findings provide new perspectives for the diagnosis and treatment of ESCC.

\section{Conclusion}

This study described, for the first time, that ETV5 promoted the progression of ESCC and ETV 5 activated MMPs through transcriptionally regulating SKA1 and TRPV2 in ESCC. We demonstrated that ETV5 expression was significantly increased in ESCC tissues, which was a risk factor for the prognosis of ESCC. Our results suggested that ETV 5 could be used as a prognostic factor and was likely to be a novel diagnostic marker and therapeutic target in ESCC treatment.

\section{Declarations}

\section{Acknowledgements}

Not applicable.

\section{Authors' contributions}

The subject design was completed by TC and MDX. The operation of the experiment was carried out by MCS, KF, and ZXL. Data analysis by LZ, ZYL, YWZ, and ZHZ. Article written by MCS and TC completed. The final article was reviewed by TC, YC, and ZYZ. All authors read and approved the final manuscript. 


\section{Funding}

This study was supported by grants from The Top-level Clinical Discipline Project of Shanghai Pudong (PWYgf2018-04), National Natural Science Foundation of China (82073224, 82072684 and 81872351) and Shanghai Committee of Science and Technology (20XD1402900, 21XD1423100, and 18140900100).

\section{Availability of data and materials}

All data generated or analyzed during this study are included in this published article.

\section{Ethics approval and consent to participate}

The animal experiment was approved and performed according to the guidelines of the Institutional Animal Care and Use Committee of Tongji University. Collection of samples with informed consent from patients and the study were approved by the Institutional Animal Care and Use Committee of Tongji University.

\section{Consent for publication}

Not applicable.

\section{Competing interests}

The authors declare no conflict of interest.

\section{References}

1. Thrift AP. Global burden and epidemiology of Barrett oesophagus and oesophageal cancer. Nature reviews Gastroenterology hepatology. 2021;18(6):432-43.

2. The global. regional, and national burden of oesophageal cancer and its attributable risk factors in 195 countries and territories, 1990-2017: a systematic analysis for the Global Burden of Disease Study 2017. The lancet Gastroenterology hepatology. 2020;5(6):582-97.

3. Arnold M, Soerjomataram I, Ferlay J, Forman D. Global incidence of oesophageal cancer by histological subtype in 2012. Gut. 2015;64(3):381-7.

4. Qi T, Qu Q, Li G, Wang J, Zhu H, Yang Z, et al. Function and regulation of the PEA3 subfamily of ETS transcription factors in cancer. American journal of cancer research. 2020;10(10):3083-105.

5. Puli OR, Danysh BP, McBeath E, Sinha DK, Hoang NM, Powell RT, et al. The Transcription Factor ETV5 Mediates BRAFV600E-Induced Proliferation and TWIST1 Expression in Papillary Thyroid Cancer Cells. Neoplasia (New York). 2018;20(11):pp. 1121-34.

6. Peng $Y$, Feng $H$, Wang $C$, Song Z, Zhang Y, Liu K, et al. The role of E26 transformation-specific variant transcription factor 5 in colorectal cancer cell proliferation and cell cycle progression. Cell death disease. 2021;12(5):427. 
7. Yoe J, Kim D, Kim S, Lee Y. Capicua restricts cancer stem cell-like properties in breast cancer cells. Oncogene. 2020;39(17):3489-506.

8. Hu D, Li Z, Li X, Fu H, Zhang M. SKA1 overexpression is associated with the prognosis of esophageal squamous cell carcinoma and regulates cell proliferation and migration. Int $\mathrm{J}$ Mol Med. 2019;44(5):1971-8.

9. Zhou K, Zhang SS, Yan Y, Zhao S. Overexpression of transient receptor potential vanilloid 2 is associated with poor prognosis in patients with esophageal squamous cell carcinoma. Medical oncology (Northwood, London, England). 2014;31(7):17.

10. He C, Wu S, Gao A, Su Y, Min H, Shang ZF, et al. Phosphorylation of ETS-1 is a critical event in DNA polymerase iota-induced invasion and metastasis of esophageal squamous cell carcinoma. Cancer Sci. 2017;108(12):2503-10.

11. Fung TM, Ng KY, Tong M, Chen JN, Chai S, Chan KT, et al. Neuropilin-2 promotes tumourigenicity and metastasis in oesophageal squamous cell carcinoma through ERK-MAPK-ETV4-MMP-E-cadherin deregulation. J Pathol. 2016;239(3):309-19.

12. Rahi A, Chakraborty M, Vosberg K, Varma D. Kinetochore-microtubule coupling mechanisms mediated by the Ska1 complex and Cdt1. Essays Biochem. 2020;64(2):337-47.

13. Huis In 't Veld PJ, Volkov VA, Stender ID, Musacchio A, Dogterom M. Molecular determinants of the Ska-Ndc80 interaction and their influence on microtubule tracking and force-coupling. eLife. 2019;8.

14. Li T, Liu X, Xu B, Wu W, Zang Y, Li J, et al. SKA1 regulates actin cytoskeleton remodelling via activating Cdc42 and influences the migration of pancreatic ductal adenocarcinoma cells. Cell proliferation. 2020;53(4):e12799.

15. Wang X, Zeng Y, Zhou M, Zhang X, Xu A, Lin J, et al. SKA1 promotes malignant phenotype and progression of glioma via multiple signaling pathways. Cancer cell international. 2019;19:324.

16. Clapham DE. TRP channels as cellular sensors. Nature. 2003;426(6966):517-24.

17. Siveen KS, Nizamuddin PB, Uddin S, Al-Thani M, Frenneaux MP, Janahi IA, et al. TRPV2: A Cancer Biomarker and Potential Therapeutic Target. Disease markers. 2020;2020:8892312.

18. Seth A, Watson DK. ETS transcription factors and their emerging roles in human cancer. European journal of cancer (Oxford, England: 1990). 2005;41(16):2462-78.

19. Zhao LJ, Yang HL, Li KY, Gao YH, Dong K, Liu ZH, et al. Knockdown of SKA1 gene inhibits cell proliferation and metastasis in human adenoid cystic carcinoma. Biomedicine pharmacotherapy $=$ Biomedecine pharmacotherapie. 2017;90:8-14.

20. Shen L, Yang M, Lin Q, Zhang Z, Miao C, Zhu B. SKA1 regulates the metastasis and cisplatin resistance of non-small cell lung cancer. Oncol Rep. 2016;35(5):2561-8.

21. Monet M, Lehen'kyi V, Gackiere F, Firlej V, Vandenberghe M, Roudbaraki M, et al. Role of cationic channel TRPV2 in promoting prostate cancer migration and progression to androgen resistance. Cancer research. 2010;70(3):1225-35. 
Figures

A

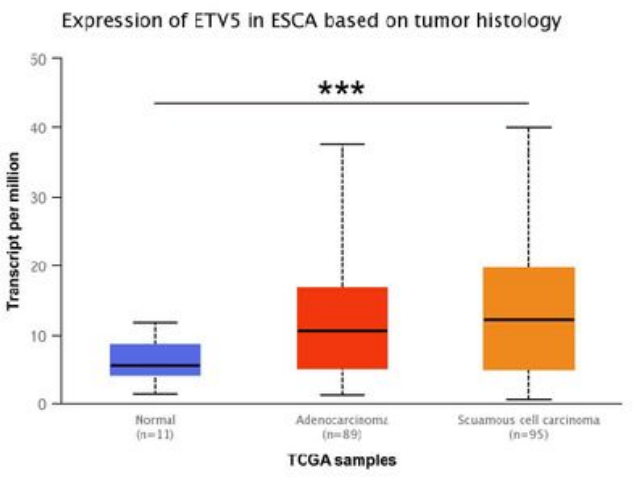

C

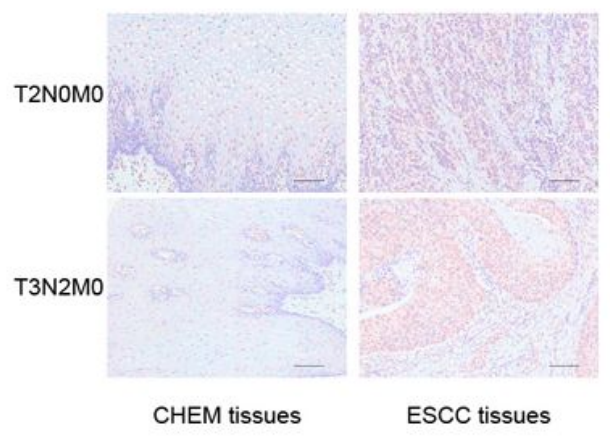

B

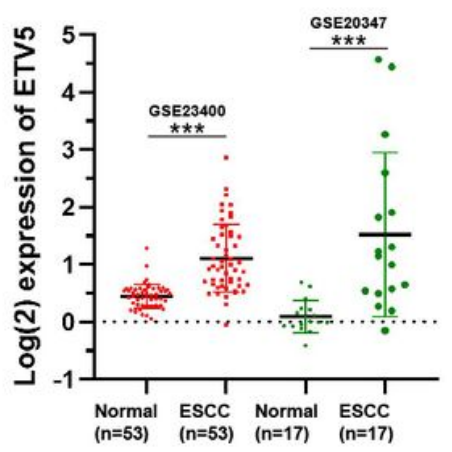

E

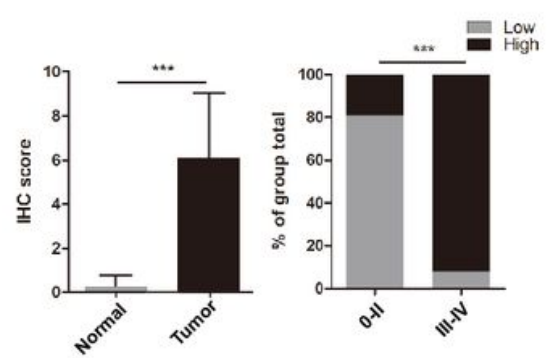

\section{Figure 1}

ETV5 is upregulated in ESCC. The level of ETV5 mRNA is upregulated in ESCC tissues indicated by data from TCGA(A) and GEO(B). (C) IHC analysis showed that ETV5 was elevated in our ESCC tissues. (D) ETV5 protein level was higher in Stage III-IV ESCC. (E) Western blot indicated that the expression level of ETV5 was higher in ESCC cells. *P囚0.05; ${ }^{\star} \mathrm{P} \otimes 0.01 ; * \star \star P \otimes 0.001$. - represents $500 \mu \mathrm{m}$. 
A
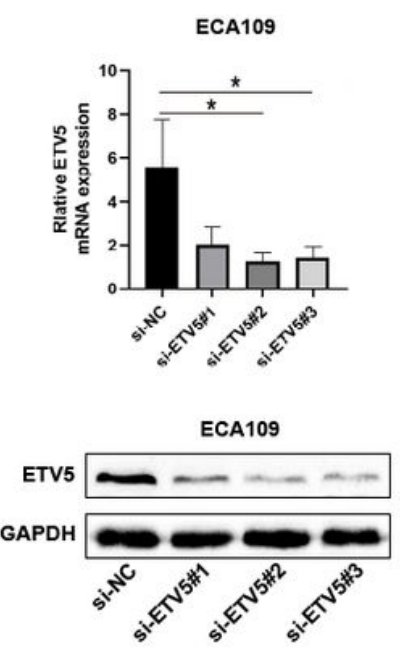

E

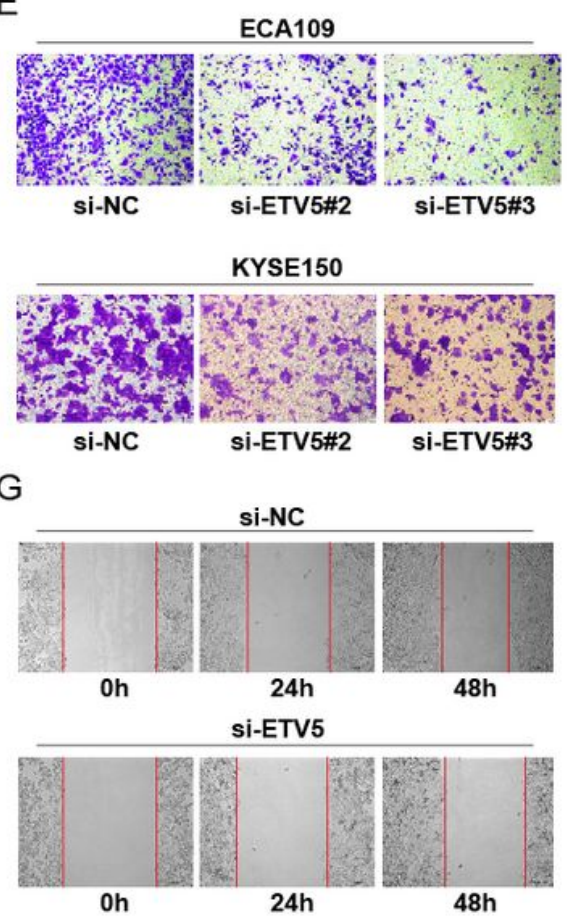

B
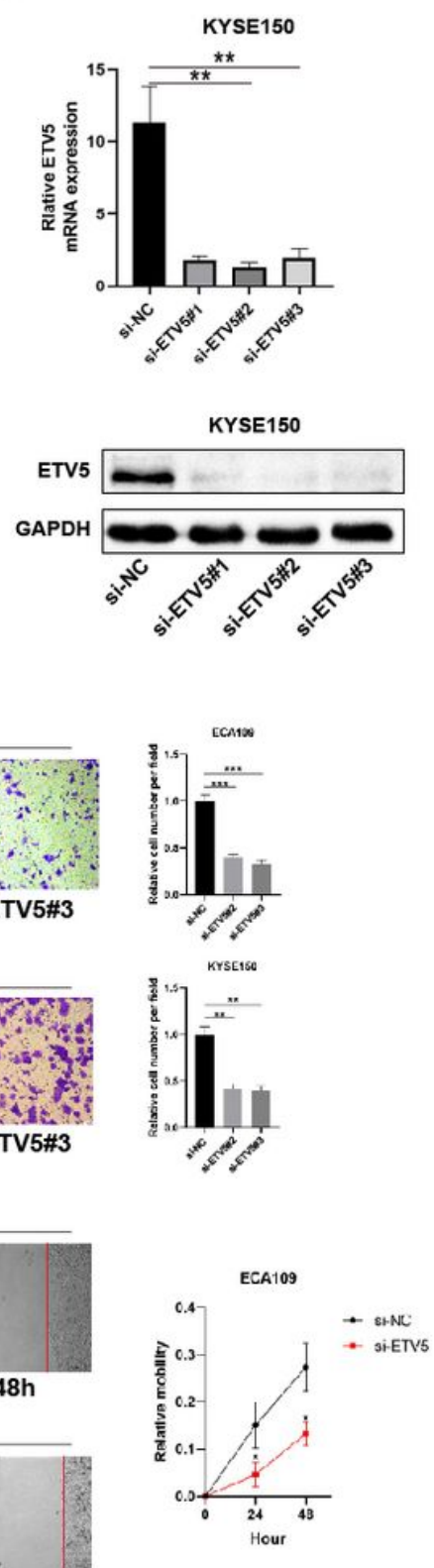

C

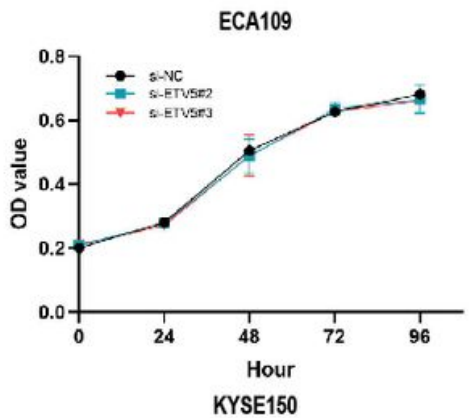

D

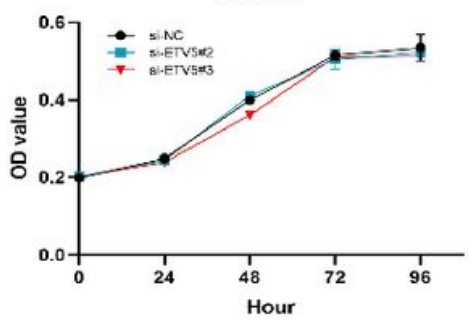

F

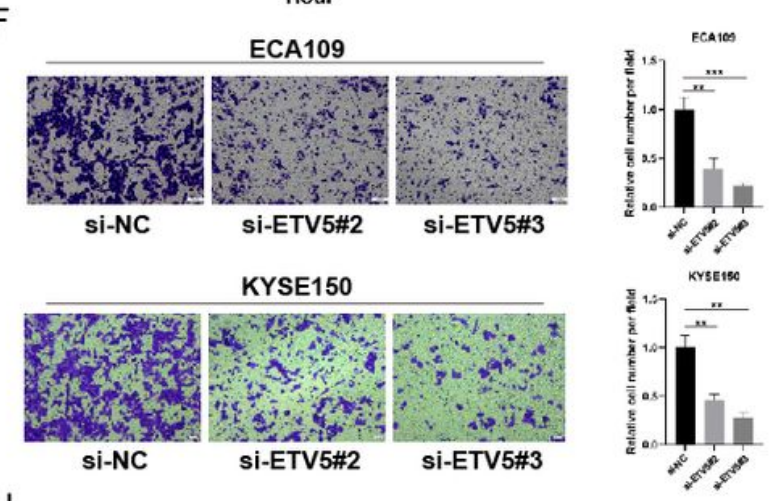

$\mathrm{H}$

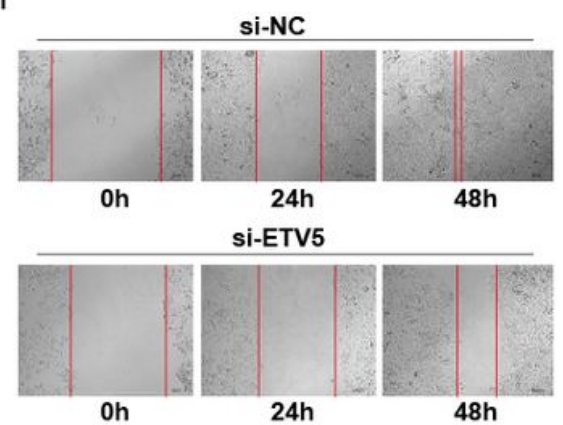

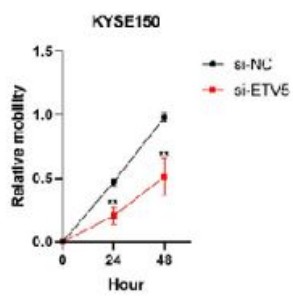

Figure 2

ETV5 affects cell migration and invasion in ESCC. (A)(B) QPCR and western blot indicated that ETV5 was significantly inhibited by siETV5 in ECA109 and KYSE150 cells. (C)(D) ETV5 knockdown did not suppress proliferation of ECA109 and KYSE150 cells, as determined via CCK8 assay. (E) ETV5 knockdown markedly inhibited migration of ECA109 and KYSE150 cells indicated by migration assay. (F) ETV5 knockdown markedly inhibited invasion of ECA109 and KYSE150 cells indicated by invasion assay. (H) ETV5 knockdown markedly suppressed migration of ECA109 and KYSE150 cells, as determined via

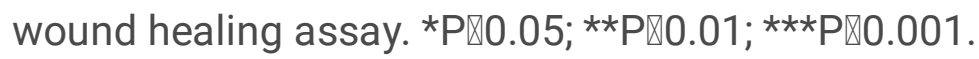


A

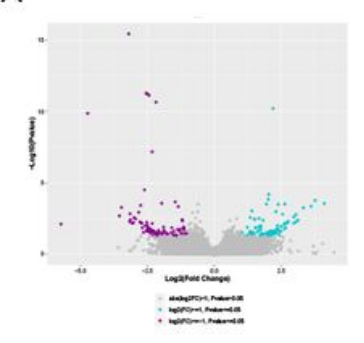

E

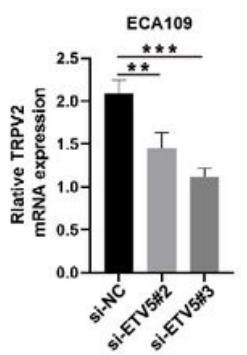

I

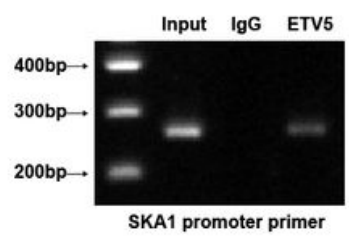

M

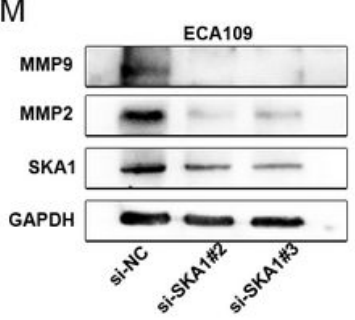

B

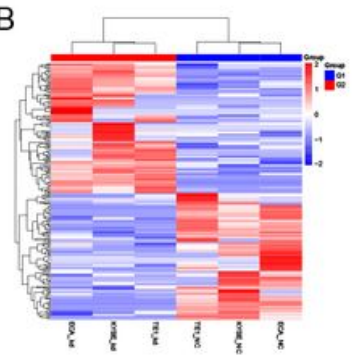

F

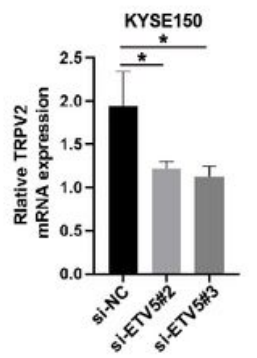

J

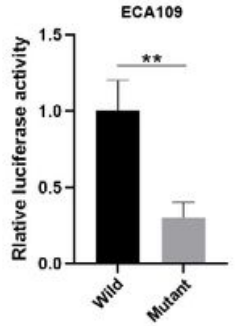

N

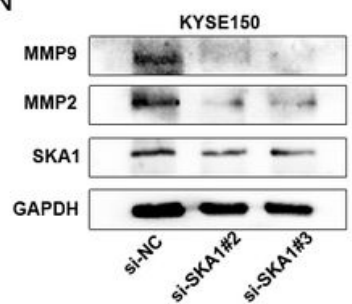

C

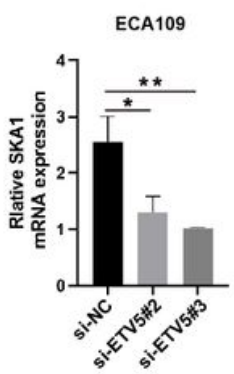

G

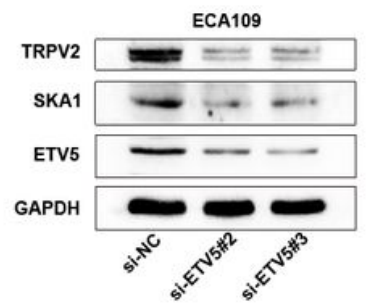

K

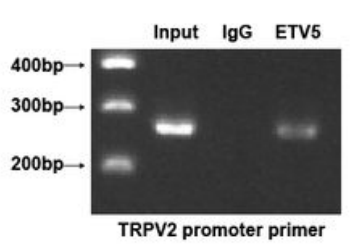

O

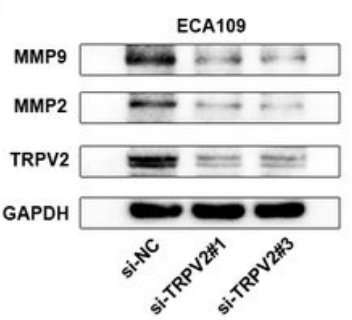

L
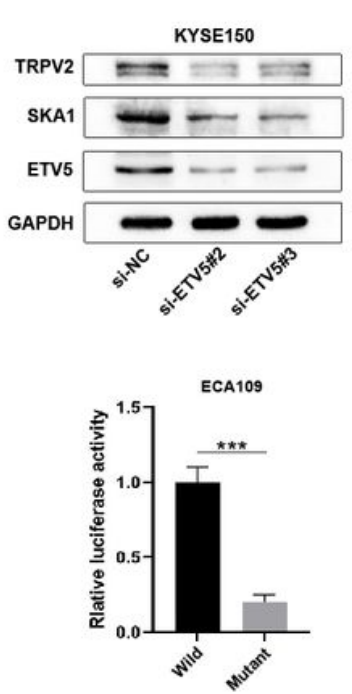

D

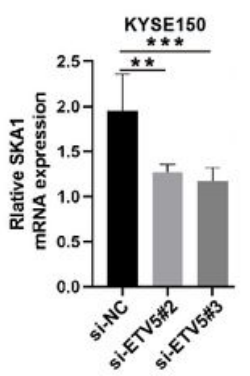

$\mathrm{H}$

P

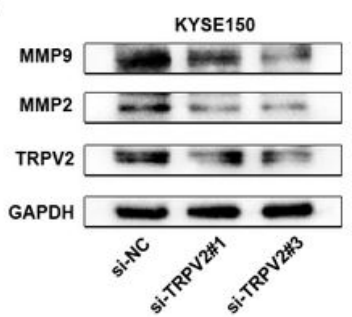

Figure 3

ETV5 activates MMPs through regulating SKA1 and further regulating TRPV2 in ESCC. (A)(B) Volcano Plot and Clustering analysis showed significantly upregulated and downregulated gene signature in ECA109, KYSE150, or TE1 siETV5 groups normalized to NC siRNA groups by RNA-seq. (C-F) QPCR indicated that the knockdown of ETV5 could downregulate SKA1 and TRPV2 mRNA expression in ECA109 and KYSE150 cells. (G)(H) Western blot showed that knockdown of ETV5 could downregulate SKA1 and TRPV2 protein expression in ECA109 and KYSE150 cells. (I) Chip assay revealed that a positive signal was detected via primers targeting SKA1. (J) Luciferase reporter gene assays showed that mutant at the predicted binding site of SKA1 significantly decreased the luciferase activity in ECA109 cells. (K) Chip assay revealed that a positive signal was detected via primers targeting TRPV2. (L) Luciferase reporter gene assays showed that mutant at the predicted binding site of TRPV2 significantly decreased the luciferase activity in ECA109 cells. (M)(N) Western blot indicated that SKA1 knockdown markedly downregulated protein expression of MMPs in EAC109 and KYSE150 cells. (O)(P) Western blot indicated 
that TRPV2 knockdown markedly downregulated protein expression of MMPs in EAC109 and KYSE150 cells. *P囚0.05; **P囚0.01; ***Pख0.001.

A
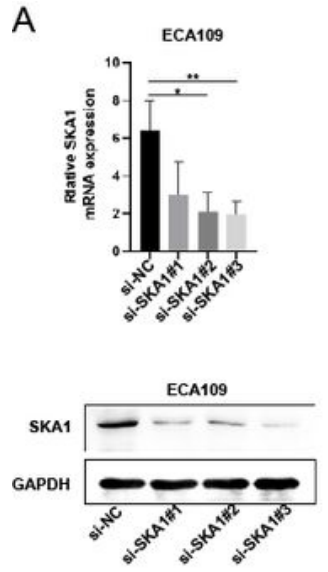

$\mathrm{E}$ 
knockdown markedly inhibited migration of ECA109 and KYSE150 cells indicated by migration assay. (F) SKA1 knockdown markedly inhibited invasion of ECA109 and KYSE150 cells indicated by invasion assay. (G) TRPV2 knockdown markedly inhibited migration of ECA109 and KYSE150 cells indicated by migration assay. (H) TRPV2 knockdown markedly inhibited invasion of ECA109 and KYSE150 cells indicated by invasion assay. (I)(J) SKA1 knockdown markedly suppressed migration of ECA109 and KYSE150 cells, as determined via wound healing assay. (K)(L) TRPV2 knockdown markedly suppressed

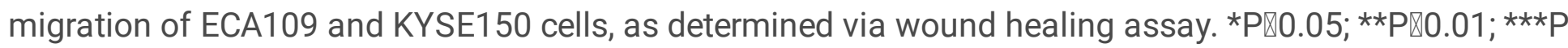
$\otimes 0.001$.

A

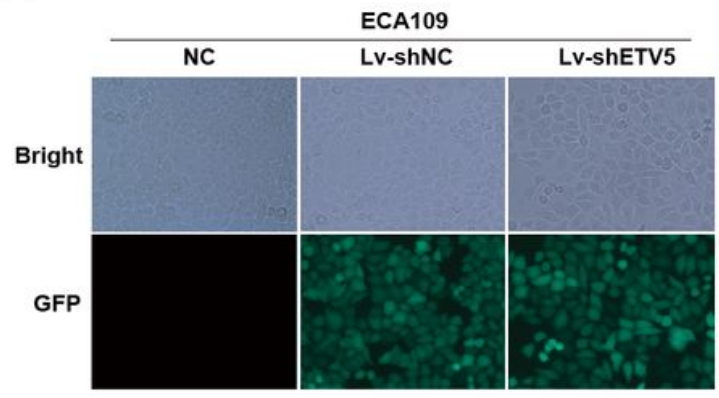

B

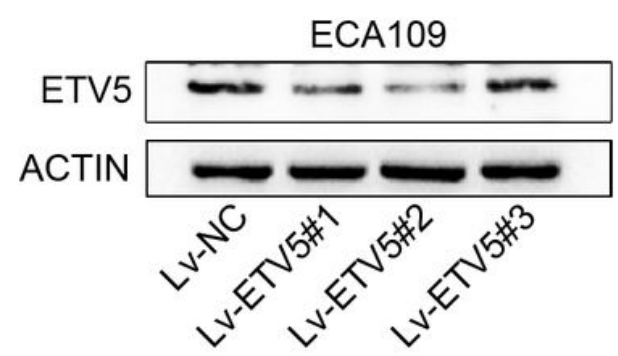

$E$
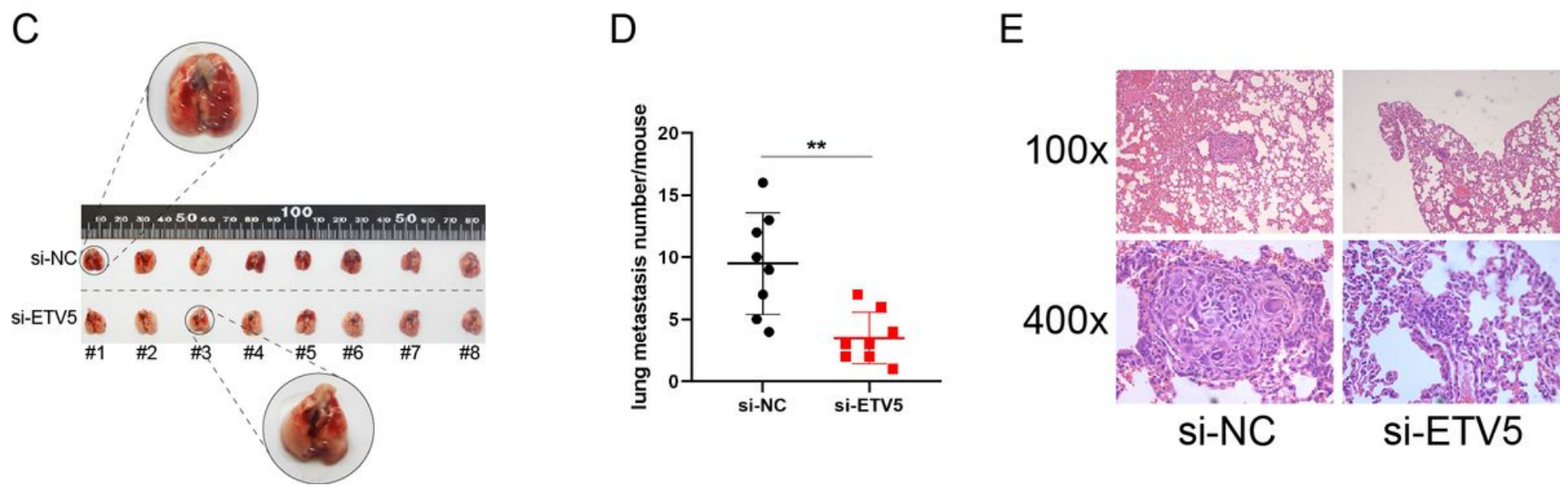

Figure 5

ETV5 affects ESCC metastasis in vivo. (A) Four days after lentivirus infection, more than $90 \%$ of ECA109 cells were GFR positive in both Lv-shRNA and LV-shETV 5 groups, as shown by fluorescence microscopy.

(B) Western blot indicated that ETV5 was significantly inhibited by LV-shETV5 in ECA109 cells. (C-E) Effects of ETV5 knockdown on colonization in the mice lungs after tail vein injection of ECA109-derived cells, including shETV5 or negative control group. Lung images were performed to detect metastasis foci (C). Metastasis quantification was evaluated (D). Representative hematoxylin and eosin (H\&E) staining of

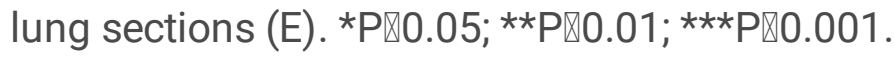




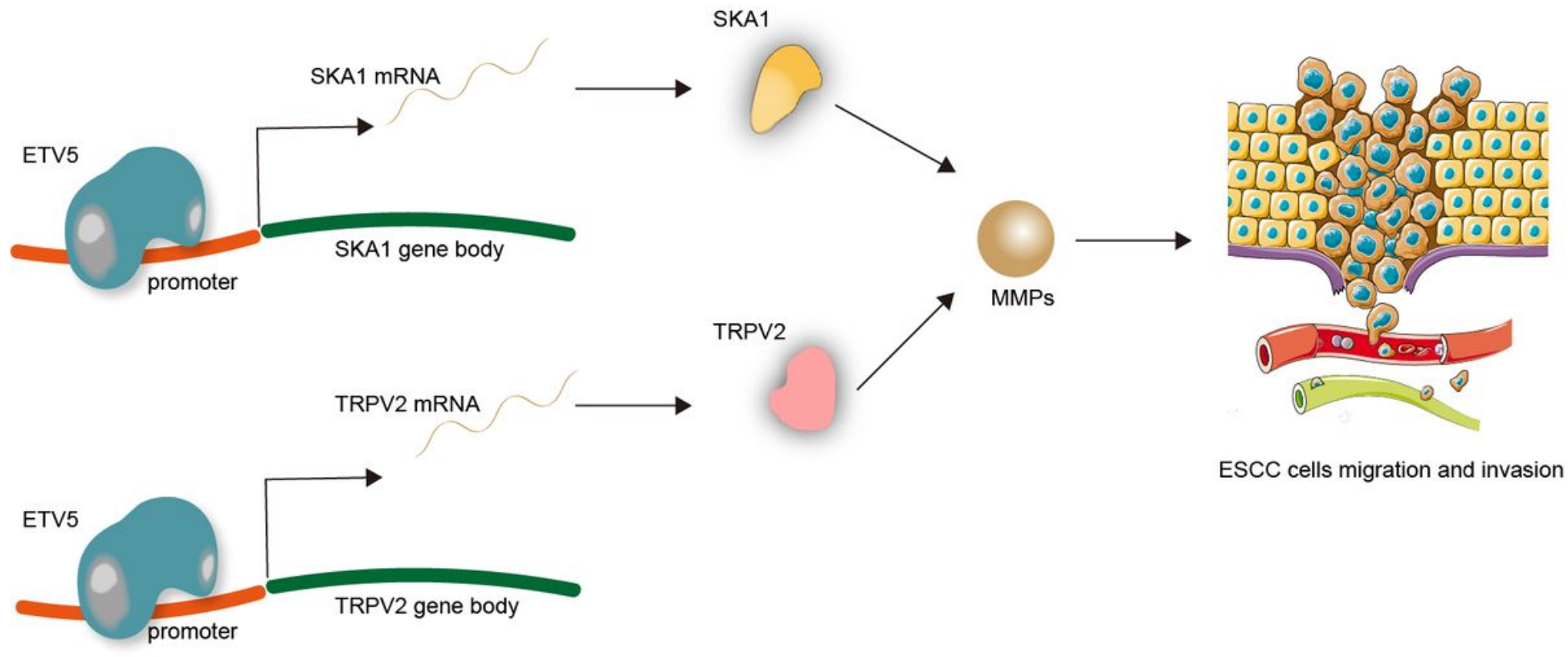

Figure 6

Schematic diagram about the underlying mechanism. 